\title{
Viabilidade e Fertilização in vitro de Oócitos Bovinos após Vitrificação
}

\author{
Viability and in vitro Fertilization of Bovine Oocytes after Vitrification
}

Sérgio Galbinski, Adriana Bos-Mikich, Arnaldo Nicola Ferrari

RESUMO

Objetivos: avaliar a técnica de criopreservação por vitrificação em DMSO 6 M para oócitos bovinos maturados in vitro e os efeitos do tempo de exposição às soluções de vitrificação (SV). Métodos: estudo experimental tipo coorte. Ovários de bovinos foram obtidos em frigorífico e transportados ao laboratório. Os oócitos foram aspirados. A partir da SV contendo DMSO $6 \mathrm{M}$ (SV 100\%), foram preparadas soluções a 25 e 65\%. Oócitos foram maturados in vitro por 1822 horas. Para vitrificação, os oócitos foram colocados em SV 25\%, por 5 minutos, transferidos à SV 65\%, pipetados em SV 100\% para palhetas e estocados em nitrogênio líquido. No primeiro grupo experimental, a exposição à SV 65\% tomou até 60 segundos e no segundo grupo não ultrapassou 30 segundos. Para descongelamento, as palhetas foram expostas ao ar por 10 segundos, colocadas em banho-maria por 10 segundos e seu conteúdo expelido e mantido em solução de sacarose por 5 minutos. No terceiro grupo, os oócitos passaram por todas SV menos pelo nitrogênio liquido. Os oócitos recuperados foram inseminados. Para controle, oócitos frescos, maturados in vitro, foram inseminados.

Resultados: após vitrificação, foram recuperados 69,1 e 59,8\% dos oócitos nos grupos de 30 segundos e 60 segundos, respectivamente, e 24 horas após inseminação pareceram morfologicamente normais 93 e 89,1\% deles, respectivamente. No grupo de oócitos expostos às $S V$ sem vitrificação, foram recuperados 75,6\%, sendo 84,6\% destes viáveis 24 horas após inseminação. Não ocorreu fertilização nos grupos experimentais. Entre os controles frescos, foram fertilizados $65,4 \%$ dos oócitos.

Conclusões: a técnica de vitrificação utilizando DMSO 6 M não é aplicável para criopreservação de oócitos bovinos maturados in vitro. A redução do tempo de exposição às SV não superou o efeito deletério sobre a capacidade fertilizadora dos oócitos. Aprimoramentos da técnica são necessários para proteção da zona pelúcida e do oolema.

PALAVRAS-CHAVE: Oócitos. Criopreservação. Fertilização in vitro. Infertilidade.

\section{Introdução}

A capacidade de criopreservar oócitos bovinos indefinidamente, para subseqüente fertilização in vitro, tem importantes aplicações na medicina humana e em biotecnologia na seleção e apri-

Fundação Universitária de Endocrinologia e Fertilidade FUEFE

Universidade Federal do Rio Grande do Sul - UFRGS

Correspondência:

Sérgio Galbinski

Rua Quintino Bocaiúva, 1035, sala 201

90440-051 - Porto Alegre - RS

Fone/Fax: (55 51) 3330-1200

e-mail:guga@orion.ufrgs.br moramento genético do gado. Em medicina humana, o desenvolvimento de técnica viável de criopreservação de oócitos poderá beneficiar pacientes previamente a tratamento oncológico, portadoras de doenças genéticas e que necessitem adiar a gestação por motivos pessoais ou profissionais. Com exceção do camundongo, no qual a criopreservação de oócitos atingiu niveis de sucesso semelhantes aos obtidos em oócitos frescos em termos de fertilização e posterior desenvolvimento embrionário ${ }^{1,2}$, o congelamento bem sucedido do gameta feminino de espécies de mamíferos ainda representa um desafio para os pesquisadores.

As duas formas mais comuns de criopreservação do gameta feminino são o congelamento lento e a vitrificação. No congelamento lento, os oócitos 
são gradativamente expostos a temperaturas cada vez mais baixas, a partir do uso de aparelho programável que realiza automaticamente a velocidade de resfriamento requerida. Na vitrificação, os gametas são expostos a temperaturas muito baixas quase imediatamente após seu contato com a solução crioprotetora e são depositados em palhetas. Ao contrário do que ocorre quando utilizado o congelamento lento, as soluções concentradas de crioprotetores, necessárias para a vitrificação, são bastante tóxicas para os gametas durante a manipulação a temperaturas acima de zero. A viabilidade e a capacidade do gameta de ser fertilizado após vitrificação são diretamente influenciadas pelo período e temperatura de manipulação dos oócitos a temperaturas acima de zero ${ }^{3,4}$.

O objetivo deste trabalho foi verificar a aplicabilidade da técnica de vitrificação utilizando uma solução $6 \mathrm{M}$ de crioprotetor dimetilsulfóxido $(\mathrm{DMSO})^{2,5}$ para a criopreservação de uma coorte de oócitos bovinos maturados in vitro para os seguintes desfechos: recuperação e sobrevivência após o descongelamento e parâmetros de viabilidade fisiológica, tais como a fecundidade e o potencial de desenvolvimento. Os objetivos específicos foram comparar o tempo de exposição às soluções de DMSO por 30 ou 60 segundos e os efeitos da exposição às soluções de DMSO sem vitrificação, com as técnicas de vitrificação, para os mesmos desfechos.

\section{Material e Métodos}

O experimento foi realizado para avaliar a sobrevivência morfológica conforme característica da zona pelúcida, retração e coloração do oócito, o potencial de fertilização e o desenvolvimento embrionário a partir de uma coorte de oócitos bovinos após vitrificação em solução $6 \mathrm{M}$ de DMSO. Também foram investigados os efeitos de toxicidade das soluções de vitrificação, sem que houvesse exposição ao nitrogênio líquido, e o efeito do tempo de exposição às soluções de vitrificação à temperatura ambiente, antes de mergulhar o material em nitrogênio líquido. Oócitos bovinos foram maturados in vitro e divididos em 6 grupos, incluindo o grupocontrole de oócitos submetidos a fertilização in vitro sem vitrificação e os 2 grupos-controle de oócitos submetidos a ativação partenogênica.

O grupo-controle de oócitos bovinos submetidos a fertilização in vitro sem vitrificação foi composto por 462 oócitos bovinos maturados in vitro e recuperados após vórtex em 10 repetições do experimento; destes, 452 foram inseminados. O grupo de oócitos bovinos submetidos a vitrificação, com exposição às soluções de vitrificação à tempera- tura ambiente por 60 segundos e posterior fertilização in vitro, foi composto por 111 oócitos bovinos maturados e recuperados após vórtex em 3 repetições do experimento. Destes, 107 (96,3\%) foram expostos às soluções de vitrificação e vitrificados em nitrogênio liquido a $-196^{\circ} \mathrm{C}$. Em outro grupo, com exposição às soluções de vitrificação à temperatura ambiente por 30 segundos e posterior fertilização in vitro, composto por 506 oócitos bovinos maturados in vitro e recuperados após vórtex em 7 repetições do experimento, 495 (97,8\%) foram expostos às soluções de vitrificação e vitrificados em nitrogênio líquido a $-196^{\circ} \mathrm{C}$. No grupo de oócitos bovinos expostos às soluções de vitrificação à temperatura ambiente por até 30 segundos, sem imersão em nitrogênio líquido e com posterior fertilização in vitro, todos 181 oócitos maturados e recuperados após vórtex em 5 repetições do experimento foram expostos às soluções de vitrificação. Destes, 137 (75,6\%) foram recuperados e inseminados. Para avaliação da ativação partenogênica 41 oócitos bovinos foram expostos a liquido seminal sem espermatozóides em 2 repetições do experimento e outros 40 oócitos foram expostos a liquido seminal com espermatozóides mortos, também em 2 repetições do experimento.

A mensuração das variáveis em estudo foi realizada em estereomicroscópio pelo mesmo pesquisador, com treinamento adequado, em todas as etapas do experimento.

A viabilidade do estudo e o tamanho da amostra foram avaliados após execução de um experimento piloto. Para uma proporção estimada de 59\% de sobrevivência após vitrificação e descongelamento e de 95\% no grupo-controle até 24 horas após a fertilização, com poder de 0,8 e alpha 0,05, seriam necessários 25 oócitos em cada grupo, perfazendo um total de 75 oócitos por experimento.

Foram coletados 461 ovários bovinos em frigoríficos oficiais logo após o abate, num total de 10 coletas. Após transporte em solução fisiológica, os folículos foram puncionados, obtendo-se 2550 oócitos, dos quais, após lavagem e seleção, conforme critérios definidos previamente, 1341 (52,5\%) foram selecionados para o experimento.

Para avaliar as proporções entre as percentagens dos dois grupos de oócitos sobreviventes a vitrificação e descongelamento, em relação ao grupo-controle submetido à fertilização in vitro e em relação ao grupo somente exposto às soluções de vitrificação sem imersão em nitrogênio líquido, foi utilizado o teste do $\chi^{2}$ de Pearson, com significância de 95\%. As probabilidades de $\mathrm{p}<0,01$ foram consideradas estatisticamente significativas.

O presente trabalho utilizou ovários obtidos a partir do abate regular de vacas em frigorificos oficiais, de acordo com as normas estaduais e fe- 
derais. Nenhum animal foi sacrificado especificamente para a realização do experimento. Nenhum animal vivo foi submetido a qualquer tipo de procedimento durante a realização do estudo. Os embriões obtidos por fertilização in vitro não foram transferidos para fêmeas receptoras. Este trabalho foi aprovado pelo Comitê de Ética em Pesquisa do Hospital de Clínicas de Porto Alegre, pela resolução número 99229.

A pesquisa foi realizada durante o período de dezembro de 1997 até dezembro de 1999. Ovários foram obtidos em frigorífico local (Frígorífico Silva, Santa Maria) e transportados ao laboratório (Laboratório de Grandes Animais da Faculdade de Medicina Veterinária da Universidade Federal de Santa Maria, RS) em solução fisiológica salina acrescida de $100 \mathrm{mg}$ de estreptomicina (Sigma, St. Louis, MO, USA) e 50.000 UI de penicilina G potássica (Sigma) à temperatura ambiente, no período máximo de 2 horas.

Os complexos cumulus-oócito (CCO) foram aspirados de folículos de 2 a $8 \mathrm{~mm}$ de diâmetro, com bomba de vácuo Nevoni sob pressão de 50 $\mathrm{mmHg}$, acoplada a uma agulha de $25 \times 7 \mathrm{G}$. Todos os CCO foram coletados em placas de Petri, sendo selecionados para maturação in vitro somente aqueles com abundantes e compactas camadas de células do cumulus.

Os CCO selecionados foram lavados três vezes em meio de maturação (TCM-199, Cultilab, Campinas, SP) acrescido de HEPES (5,95 mg/mL; Sigma), piruvato de sódio $(0,025 \mathrm{mg} / \mathrm{mL}$; Sigma), bicarbonato de sódio $(2,2 \mathrm{mg} / \mathrm{mL}$; Sigma), hormônio folículo-estimulante recombinante $(\mathrm{r}-\mathrm{FSH}$; 0,01 UI/mL; Serono, Itália), hormônio luteinizante (LH; $0,5 \mu \mathrm{g} / \mathrm{mL}$; Serono) e $10 \%$ de soro de vaca em estro preparado no próprio laboratório, à temperatura de 25 a $30^{\circ} \mathrm{C}$. Grupos de 20 a 30 CCO foram depositados em gotas de $400 \mu \mathrm{L}$ de meio de maturação em placas de cultura, cobertas de óleo mineral (Sigma) a $39^{\circ} \mathrm{C}$, em atmosfera de $5 \% \mathrm{CO}_{2}$, por períodos entre 18 e 22 horas.

A solução de vitrificação de estoque (SV $111 \%$ ) consistiu de DMSO $6 \mathrm{M}$ em meio Ham F10. Esta solução foi mantida em geladeira a $4^{\circ} \mathrm{C}$ até o uso. A SV 100\% foi preparada a partir da adição de $2 \mathrm{~mL}$ de soro fetal bovino (SFB; Cultilab, Campinas) a $18 \mathrm{~mL}$ de SV $111 \%$. Diluições da SV $100 \%$ foram preparadas de modo a resultar em SV 65\% (3,9 M de DMSO) e em SV 25\% (1,5 M de DMSO). A solução de sacarose $1 \mathrm{M}$ foi preparada pela adição de $1,71 \mathrm{~g}$ de sacarose (Sigma) em $5 \mathrm{~mL}$ de meio Ham F-10 acrescido de $10 \%$ de SFB.

As palhetas plásticas de 0,25 mL (Nutricell, Campinas, Brasil) foram preparadas com o tampão de algodão cerca de $4 \mathrm{~cm}$ no seu interior, com uma coluna de $3 \mathrm{~cm}$ da solução de sacarose $1 \mathrm{M}$ molhando o tampão e com uma fração de $1 \mathrm{~cm}$ de SV $100 \%$ depositada na palheta, permanecendo isolada da gota de sacarose por uma bolha de ar. Após maturação e remoção do excesso de células do cumulus com uso de vórtex com $60 \%$ de velocidade, durante 1 minuto, grupos de $10 \mathrm{CCO}$ foram expostos à SV 25\%, à temperatura ambiente por 5 minutos, e então transferidos para $1 \mathrm{~mL}$ da solução SV $65 \%$, com um mínimo volume da SV $25 \%$ e misturados completamente. Os CCO foram transferidos com um minimo volume de SV $65 \%$ para a coluna de SV $100 \%$ dentro da palheta. Esta foi selada com massa de hematócrito e exposta ao vapor de nitrogênio líquido por 3 minutos antes de ser imersa e vitrificada. As palhetas foram armazenadas por períodos que variaram de 4 horas a 3 meses. Os procedimentos de colocação dos CCO em SV 65\% e de exposição ao vapor de nitrogênio liquido demoraram até 60 segundos no primeiro grupo experimental e até 30 segundos no segundo grupo experimental.

Grupos de 10 CCO foram equilibrados na SV $25 \%$ por 5 minutos, lavados em SV $65 \%$ e colocados nas palhetas contendo SV $100 \%$, as quais foram seladas e manipuladas como descrito acima, mas sem exposição ao vapor e imersão no nitrogênio líquido. Todo o processo entre a SV 65\% e a SV 100\% não ultrapassou 30 segundos de duração. Logo a seguir, as palhetas foram descarregadas em solução de sacarose e processadas para remoção do crioprotetor, conforme descrito abaixo para as amostras vitrificadas.

As amostras vitrificadas foram reaquecidas por exposição à temperatura ambiente (em torno de $20^{\circ} \mathrm{C}$ ) por 10 segundos e transferidas para banhomaria a $25^{\circ} \mathrm{C}$ por 10 segundos. Os conteúdos de cada palheta foram expelidos em um vidro de relógio contendo $1 \mathrm{~mL}$ de solução de sacarose $1 \mathrm{M}$ à temperatura ambiente, misturados e, neste local, mantidos por 5 minutos em sacarose. Os oócitos foram transferidos para $1 \mathrm{~mL}$ de meio de fertilização (Fert-talp) ${ }^{6}$, lavados duas vezes e mantidos em $400 \mu \mathrm{L}$ deste meio por 2 horas antes da inseminação.

Amostras de sêmen congelado foram utilizadas para fertilização in vitro. O sêmen congelado foi descongelado em banho-maria a $39^{\circ} \mathrm{C}$, por 10 segundos. Foram depositados $100 \mu \mathrm{L}$ de cada amostra sob $1 \mathrm{~mL}$ de meio Sperm-talp ${ }^{6}$ em tubos cônicos que foram colocados em banho-maria a $39^{\circ} \mathrm{C}$ por 1 hora. O sobrenadante foi então transferido para tubos de centrifuga e centrifugado por 10 minutos. Após remoção do sobrenadante, o pellet foi recolocado em meio Sperm-talp de maneira a obter-se concentração final de $1 \times 10^{6}$ espermatozóides/mL.

Para fertilização in vitro dos oócitos, $\mathrm{CCO}$ maturados in vitro foram colocados em $400 \mu \mathrm{L}$ do meio Fert-talp ${ }^{6}$, sob óleo, e mantidos a $39^{\circ} \mathrm{C}$ em atmosfera de $5 \%$ de $\mathrm{CO}_{2}$ até o momento da inseminação. Foram adicionados $2 \mu \mathrm{L}$ de heparina 
e $50 \mu \mathrm{L}$ de suspensão de esperma à gota contendo ambos os grupos de CCO, frescos e vitrificados/ descongelados. O sistema foi mantido em atmosfera de $\mathrm{CO}_{2}$, a $39^{\circ} \mathrm{C}$, por 18 a 22 horas.

Após a fertilização in vitro, o excesso de células dos CCO foi removido por vórtex com $60 \%$ de velocidade durante 1,5 minuto. Os oócitos, fecundados ou não, foram lavados duas vezes e transferidos para meio de cultivo embrionário $\mathrm{CR} 1^{6}$, em atmosfera de $5 \%$ de $\mathrm{CO}_{2}$, a $39^{\circ} \mathrm{C}$. A avaliação após 24 horas considerou viáveis os oócitos que não apresentavam retração e/ou granulação, que apresentavam membrana integra e sem escurecimento. Eles foram cultivados por 48 horas, para posterior avaliação da taxa de fertilização e desenvolvimento embrionário. As taxas de fertilização foram calculadas como o número de embriões de 2 células presentes após 48 horas de cultivo. A cultura embrionária procedeu até o $7^{\circ}$ dia para constatação da freqüência de mórulas e blastocistos.
Para avaliação de possível ativação partenogênica dos oócitos durante a FIV, um grupo de $\mathrm{CCO}$ frescos foi exposto a fluido seminal com espermatozóides mortos e outro grupo foi exposto a fluido seminal sem espermatozóides.

\section{Resultados}

A fertilização in vitro e o desenvolvimento embrionário de 452 oócitos frescos foram determinados neste experimento (Tabela 1). A maioria $(94,6 \%)$ dos oócitos inseminados foram considerados morfologicamente normais 24 horas após a inseminação. A taxa de fertilização avaliada como o número de embriões que atingiram o estágio de 2 células 48 horas após inseminação foi de $65,4 \%$. Dos embriões obtidos, $28,2 \%$ evoluíram ao estágio de blastocisto até o sétimo dia.

Tabela 1 - Resultados da fertilização in vitro e desenvolvimento embrionário de oócitos bovinos frescos

\begin{tabular}{lccccc}
\hline & Oócitos maturados & Oócitos inseminados & $\begin{array}{c}\text { Oócitos viáveis } \\
\text { pós-inseminação }\end{array}$ & Embriões clivados & $\begin{array}{c}\text { Blastocistos } \\
\text { (7- dia) }\end{array}$ \\
\hline Número & 462 & 452 & 428 & 280 & 79 \\
$\%$ & - & - & 94,6 & $65,4^{1}$ & $28,2^{2}$ \\
& & & & & $18,4^{1}$ \\
\hline
\end{tabular}

$\%$ sobre o total de oócitos inseminados.

$2 \%$ sobre o número de embriões obtidos.

Foram expostos 107 CCO às SV 65 e 100\% por 60 segundos à temperatura ambiente, antes da exposição ao vapor e imersão no nitrogênio líquido para vitrificação. Após o descongelamento, 64 oócitos $(59,8 \%)$ foram recuperados e submetidos à inseminação. Não ocorreu fertilização nem desenvolvimento embrionário neste grupo. A viabilidade morfológica, 24 horas pós-inseminação, não diferiu significativamente daquela do grupocontrole $(p=0,089)$ (Tabela 2).

Tabela 2 - Resultados de viabilidade e fertilização in vitro após vitrificação de oócitos bovinos utilizando diferentes tempos de exposição às soluções de vitrificação (SV).

Número de

Tempo de exposição

(segundos)

\begin{tabular}{lcc} 
& 30 & 60 \\
\hline Oócitos expostos às SV & 495 & 107 \\
Oócitos recuperados pós-vitrificação & $342(69,1 \%)^{1}$ & $64(59,8 \%)^{1}$ \\
Oócitos viáveis pós-inseminação & $319(93 \%)^{2}$ & $57(89,1 \%)^{2}$ \\
Embriões clivados & - & - \\
\hline
\end{tabular}

$1 \%$ sobre o número total de oócitos vitrificados ( $\mathrm{p}=0,493$ ).

$2 \%$ sobre o número de oócitos recuperados pós-descongelamento $(p=0,293)$.
Foram expostos 495 CCO às SV 65 e 100\% por 30 segundos à temperatura ambiente, antes de serem expostos ao vapor e imersos no nitrogênio líquido para vitrificação. Após o descongelamento foram recuperados 342 oócitos $(69,1 \%)$, os quais foram submetidos à fertilização in vitro. Vinte e quatro horas após a inseminação, 319 (93\%) pareceram morfologicamente normais. Não foi detectada clivagem 48 horas após inseminação. Não houve diferença significativa entre a taxa de sobrevivência do presente grupo e a taxa do grupo exposto às SV por até 60 segundos $(p=0,293)$, assim como em relação ao grupo controle $(p=0,493)$ (Tabela 2).

Um total de $181 \mathrm{CCO}$ foram expostos por até 30 segundos às SV 65 e 100\%, à temperatura ambiente e imediatamente expelidos na solução $1 \mathrm{M}$ de sacarose para remoção do crioprotetor sem vitrificação. Destes, 137 (75,6\%) foram recuperados após a exposição, dos quais $116(84,6 \%)$ foram considerados morfologicamente normais 24 horas após a inseminação. Não ocorreu fertilização nem desenvolvimento embrionário neste grupo. A taxa de sobrevivência, analisada 24 horas após a 
inseminação, foi significativamente diferente entre o presente grupo e os controles $(p<0,001)$, assim como em relação ao grupo de exposição por até 30 segundos $(p=0,006)$. Não houve diferença significativa nas taxas de viabilidade deste grupo e o grupo com exposição por até 60 segundos $(p=0,293)($ Tabela 3$)$.

Cada experimento de partenogênese utilizou um total de $40 \mathrm{CCO}$ frescos, não tendo sido detectada qualquer clivagem.

Tabela 3 - Resultados de viabilidade e fertilização in vitro de oócitos após exposição às soluções de vitrificação, sem vitrificação.

\begin{tabular}{lcccc}
\hline & Maturados & Recuperados pós-exposição & Viáveis pós-inseminação & Embriões clivados \\
\hline Número & 181 & 137 & $116^{1}$ & - \\
$\%$ & & 75,6 & $84,6^{1}$ & - \\
\hline
\end{tabular}

${ }^{1}$ significativamente diferente do grupo controle $(p<0,001)$ e em relação ao grupo de oócitos vitrificados com exposição às soluções de vitrificação por até 30 segundos $(p=0,006)$.

\section{Discussão}

Nossos resultados demonstram que a vitrificação em DMSO $6 \mathrm{M}$ não é técnica apropriada para a criopreservação de oócitos bovinos. A fertilização e o desenvolvimento embrionário não foram possiveis após vitrificação ou exposição às soluções de vitrificação em quaisquer dos grupos experimentais.

O processo de vitrificação empregado em nosso estudo foi escolhido para criopreservar oócitos bovinos por seus excelentes resultados com oócitos de camundongo e de hamster ${ }^{2,5}$. Os bovinos, por serem grandes mamíferos, são modelos animais mais próximos dos humanos. Entretanto, o mesmo sucesso não foi atingido para a espécie bovina, devido à total ausência de fertilização e de desenvolvimento embrionário após descongelamento ou exposição às soluções de vitrificação.

De forma geral, a recuperação de oócitos com uma morfologia aparentemente normal, neste trabalho, foi elevada, e esta observação nos leva a sugerir efeito deletério na zona pelúcida ou no oolema, que impediu a fertilização. O problema da falha de fertilização observado com freqüência nos primeiros experimentos de criopreservação de oócitos mamíferos foi associado a alterações que ocorrem na zona pelúcida ${ }^{2,7}$. O problema foi superado no camundongo pela adição de SFB aos meios de congelamento e de diluição ${ }^{1}$. Neste estudo, oócitos bovinos foram vitrificados em meio contendo $10 \%$ de SFB, entretanto, houve falha permanente da fertilização. Talvez, a concentração de $10 \%$ de SFB seja insuficiente para proteger a zona pelúcida dos oócitos bovinos durante a exposição, apesar de breve, às altas concentrações de crioprotetores. Alternativamente, podem ocorrer variações dos componentes do SFB que são necessários para proteger os oócitos bovinos. Em seu estudo, Wood et al. ${ }^{2}$ notaram que, quando os oócitos de hamster eram vi- trificados utilizando SFB chileno, era necessário aumentar sua concentração a $15 \%$, de maneira a obterem-se taxas de sobrevivência semelhantes àquelas quando se utilizava o SFB inglês na concentração de $5 \%$.

Para diminuir o efeito citotóxico das altas concentrações de crioprotetores necessárias para vitrificação, tem sido sugerida a redução do tempo de exposição aos crioprotetores necessário para vitrificação ${ }^{8}$, sua exposição apenas a baixas temperaturas $^{9}$ e a diminuição da concentração de crioprotetores ou sua mistura com outros menos tóxicos ${ }^{9-11}$.

No presente estudo, uma modificação do procedimento original de Wood et $\mathrm{al}^{2}$ foi pesquisada para reverter o efeito deletério na fertilização. Foi experimentada redução no tempo de exposição às SV à temperatura ambiente, antes de colocar os oócitos nas palhetas. Com isto, procuramos diminuir os efeitos potencialmente deletérios da toxicidade química causada pelo DMSO à temperatura ambiente. Entretanto, esta modificação não levou a qualquer melhora na capacidade dos oócitos vitrificados de serem fertilizados após o descongelamento. Algumas técnicas para redução do tempo de exposição utilizando grids metálicos, de náilon ou palhetas abertas obtiveram algum sucesso, inclusive em oócitos humanos, mas são pouco viáveis para utilização em larga escala ${ }^{12,13}$.

Consideradas em conjunto, nossas observações sugerem que a falha total da fertilização foi provavelmente causada por modificações estruturais na zona pelúcida devido a alterações nos receptores de espermatozóide ZP2 e ZP2f, durante a exposição às SV, conforme já foi relatado anteriormente para o oócito humano e de coelho ${ }^{13,14}$. Mais recentemente foi relatado que o resfriamento de oócitos bovinos maturados in vitro diminuiria as taxas de oócitos fertilizados e o desenvolvimento embrionário in vitro posterior, parcialmente devido a defeitos na zona pelúcida ${ }^{15,16}$. 
Por outro lado, podem ter ocorrido danos à membrana plasmática, os quais poderiam, talvez, ser evitados pelo emprego de método de equilíbrio em múltiplas etapas, conforme descrito por Kuwayama et al. ${ }^{17}$, para a vitrificação de blastocistos bovinos. O trabalho daqueles autores demonstrou que a criopreservação de blastocistos, conforme o método de equilíbrio em 16 etapas, diminui os danos ultraestruturais à membrana plasmática observados quando o método de duas etapas é empregado. Os efeitos do resfriamento na integridade da membrana dos oócitos bovinos também puderam ser superados pelo seu tratamento com hidroxitolueno butilado antes da exposição a temperaturas mais baixas ${ }^{18}$.

A adição de citocalazina $\mathrm{D}$ à solução de vitrificação não melhorou os resultados ${ }^{19}$. Crioprotetores como DAP 213 induziram alterações nos microtúbulos e nas mitocôndrias, causando liberação precoce dos grânulos corticais e ausência de clivagem ${ }^{20}$. Recentemente foi proposto que a citocalazina $\mathrm{D}$ poderia proteger os microtúbulos, mas sem proteção á mitocôndria e sem melhora significativa dos resultados ${ }^{21}$. A técnica de vitrificação com uma solução que inclui o crioprotetor etilenoglicol a $20 \%$ tem resultado em taxas de clivagem de 38 a 49\%, o que aparentemente é promissor $^{19,22}$.

Qualquer que seja o local do dano causado pelo método de vitrificação aqui empregado, seja na zona pelúcida ou na membrana plasmática, alternativa para se avaliar a viabilidade do oócito seria a injeção intracitoplasmática de espermatozóides (ICSI) nos oócitos sobreviventes à exposição às soluções de vitrificação e ao congelamento, o que já foi realizado inclusive para oócitos humanos, com nascimento-vivo ${ }^{23}$. Não executamos ICSI no presente estudo devido a limitações técnicas, mas é nosso objetivo introduzir esta técnica em nossos futuros experimentos de criopreservação.

Em conclusão, a vitrificação de oócitos bovinos utilizando a solução de DMSO $6 \mathrm{M}$, conforme descrita para o oócito murino, não é procedimento válido. A diminuição no tempo de exposição às SV à temperatura ambiente não superou os efeitos deletérios causados pela técnica na capacidade dos oócitos vitrificados e descongelados de serem fertilizados e no seu desenvolvimento embrionário. Entretanto, modificações da técnica original podem levar a resultados positivos para a criopreservação do gameta feminino bovino por vitrificação.

\section{ABSTRACT}

Purpose: to verify vitrification techniques using $6 \mathrm{M} D \mathrm{DMSO}$ to cryopreserve in vitro matured bovine oocytes, and to assess the effects of the time of exposure to vitrification solutions (VS).

Methods: dilutions of VS were prepared from the stock VS (VS 100\%) consisting of $6 \mathrm{M}$ DMSO to give 25 and $65 \%$ DMSO solutions. Bovine oocytes were in vitro matured for 18-22 h. Matured oocytes were placed first into $25 \% \mathrm{VS}$, at room temperature for $5 \mathrm{~min}$, then transferred to $65 \% \mathrm{VS}$, before being pipetted into the $100 \%$ VS in plastic straws. Three experimental groups were formed: in the first group, time of pipetting through $65 \%$ VS and loading the straw took up to $60 \mathrm{~s}$, in the second group it did not exceed $30 \mathrm{~s}$. For thawing, straws were held in air for $10 \mathrm{~s}$ and then in a water bath for $10 \mathrm{~s}$. The contents of each straw were expelled in sucrose solution and held for $5 \mathrm{~min}$. In the third experimental group, oocytes went through all VS, but were not vitrified. All retrieved oocytes were inseminated. For control, fresh, in vitro matured oocytes were inseminated. Results: after vitrification, 69.1 and $59.8 \%$ of the oocytes were retrieved from the $30 \mathrm{~s}$ and $60 \mathrm{~s}$ groups, respectively, and 93 and $89 \%$ of these oocytes appeared morphologically normal $24 \mathrm{~h}$ after insemination, respectively. In the group of oocytes exposed without vitrification, $75.6 \%$ were retrieved and $84.7 \%$ were morphologically viable, $24 \mathrm{~h}$ after insemination. No fertilization was observed in the experimental groups. Among controls, 65.4\% were fertilized. Conclusions: the vitrification technique using 6 M DMSO is not a feasible approach to cryopreserve in vitro matured bovine oocytes. Decreasing the time of exposure to VS did not overcome deleterious effects of the procedure on the fertilizability of oocytes. Improvements in the technique are needed to protect the zona pellucida and oolemma.

KEYWORDS: Oocyte. Cryopreservation. Vitrification. Fertilization.

\section{Referências}

1. Carroll J, Wood MJ, Whittingham DG. Normal fertilization and development of frozen-thawed mouse oocytes: protective action of certain macromolecules. Biol Reprod 1993; 48:606-12.

2. Wood MJ, Barros C, Candy CJ, Carroll J, Melendez $\mathrm{J}$, Whittingham DG. High rates of survival and fertilization of mouse and hamster oocytes after vitrification in dimethylsulphoxide. Biol Reprod 1993; 49:489-95.

3. Rall WF, Fahy GM. Ice-free cryopreservation of mouse embryos at $-196^{\circ} \mathrm{C}$ by vitrification. Nature 1985; 313:573-5. 
4. Kono T, Kwon OY, Nakahara T. Development of vitrified mouse oocytes after in vitro fertilization. Cryobiology 1991; 28:50-4.

5. Bos-Mikich A, Wood MJ, Whittingham DG. Cytogenetical analysis and developmental potential of vitrified mouse oocytes. Biol Reprod 1995; 53:780-5.

6. Parrish JJ, Susko-Parrish JL, First NL. In vitro fertilization of bovine oocytes using heparin treated and swim-up separated frozen-thawed bovine semen is repeatable and results in high frequencies of fertilization. Theriogenology 1985; 23:216-22.

7. Carroll J, Depypere H, Matthews CD. Freeze-thawinduced changes of the zona pellucida explains decreased rates of fertilization in frozen-thawed mouse oocytes. J Reprod Fertil 1990; 90:547-53.

8. Arav A, Rubinsky B, Fletcher G, Seren E. Cryogenic protection of oocytes with antifreeze proteins. Mol Reprod Dev 1993; 36:488-93.

9. Rall WF. Factors affecting the survival of mouse embryos cryopreserved by vitritication. Cryobiology $1987 ; 24: 387-402$.

10.Massip A, Van der Zwalmen P, Scheffen B, Ectors F. Pregnancies following transfer of cattle embryos preserved by vitrification. Cryo Letters 1986; 7:270-3.

11.Dhali A, Manik RS, Das SK, Singla SK, Palta P. Vitrification of buffalo (Bubalus bubalis) oocytes. Theriogenology 2000; 53:1295-303.

12.Vajta G, Holm P, Kuwayama M, et al. Open Pulled Straw (OPS) vitrification: a new way to reduce cryoinjuries of bovine ova and embryos. Mol Reprod Dev 1998; 51:53-8.

13.Wu J, Zhang L, Wang X. In vitro maturation, fertilization and embryo development after ultrarapid freezing of immature human oocytes. Reproduction 2001; 121:389-93.
14. Sathananthan AH, Trounson A, Freeman L. Morphology and fertilizability of frozen human oocytes. Gamete Res 1987; 16:343-54.

15.Vincent C, Turner K, Pickering SJ, Johnson MH. Zona pellucida modifications in the mouse in the absence of oocyte activation. Mol Reprod Dev 1991; 28:394-404.

16.Azambuja RM, Kraemer DC, Westhusin ME. Effect of low temperatures on in vitro matured bovine oocytes. Theriogenology 1998; 49:1155-64.

17.Kuwayama M, Fujikawa S, Nagai T, Ultrastructure of IVM-IVF bovine blastocysts vitrified after equilibration in glycerol 1,2-propanediol using 2step and 16-step procedures. Cryobiology 1994; 31:415-22.

18.Zeron Y, Pearl M, Borochov A, Arav A. Kinetic and temporal factors influence chilling injury to germinal vesicle and mature bovine oocytes. Cryobiology 1999; 38:35-42.

19.Vieira AD, Mezzalira A, Barbieri DP, Lehmkuhl RC, Rubin MI, Vajta G. Calves born after open pulled straw vitrification of immature bovine oocytes. Cryobiology 2002; 45:91-4.

20.Fuku EJ, Liu J, Downey BR. In vitro viability and ultrastructural changes in bovine oocytes treated with a vitrification solution. Mol Reprod Dev 1995; 40:177-85.

21.Rho GJ, Kim S, Yoo JG, Balasubramanian S, Lee HJ, Choe SY. Microtubulin configuration and mitochondrial distribution after ultra-rapid cooling of bovine oocytes. Mol Reprod Dev 2002; 63:464-70.

22.Lj X, Su L, Li Y, Ji W, Dinnyes A. Vitrification of Yunnan Yellow Cattle oocytes: work in progress. Theriogenology 2002; 58:1252-60.

23.Kuleshova L, Gianaroli L, Magli C, Ferraretti A, Trounson A. Birth following vitrification of a small number of human oocytes: case report. Hum Reprod 1999; 14:3077-9.

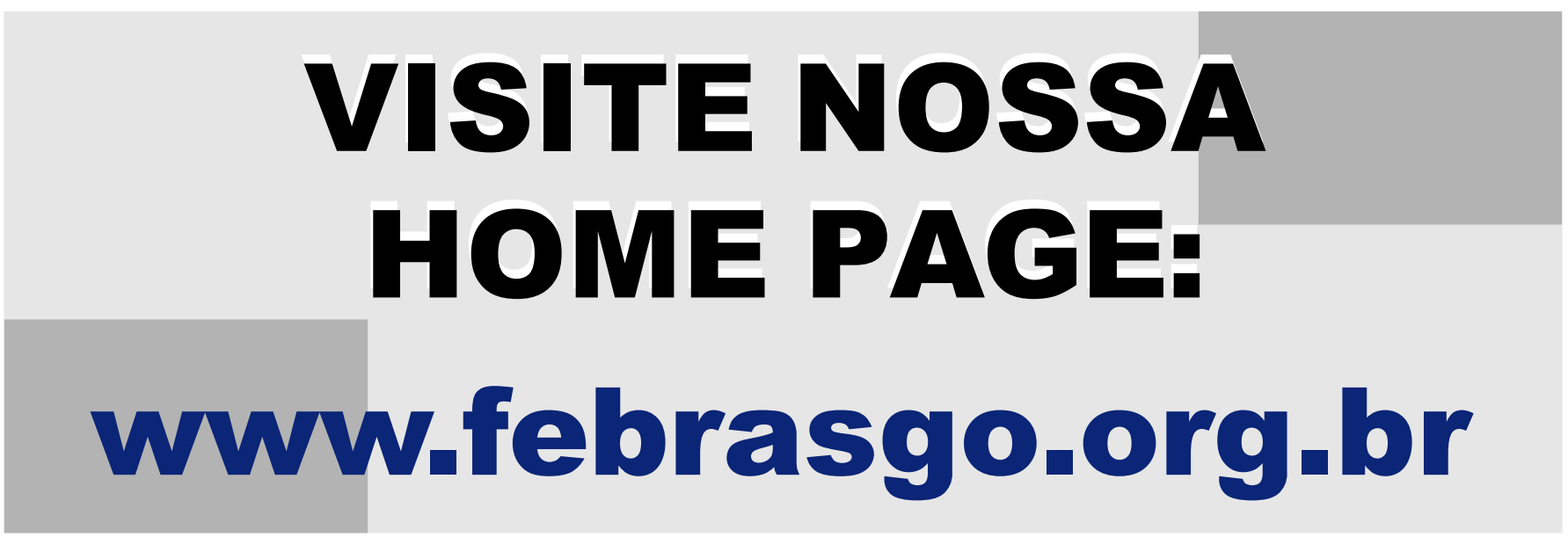

